\title{
German language questionnaires for assessing implementation constructs and outcomes of psychosocial and health- related interventions: a systematic review
}

\author{
Christina Kien ${ }^{1,2^{*}}$, Marie-Therese Schultes ${ }^{3,4}$, Monika Szelag ${ }^{1}$, Rudolf Schoberberger ${ }^{2}$ and Gerald Gartlehner ${ }^{1,5}$
}

\begin{abstract}
Background: Over the past years, implementation science has gained more and more importance in German-speaking countries. Reliable and valid questionnaires are needed for evaluating the implementation of evidence-based practices. On an international level, several initiatives focused on the identification of questionnaires used in English-speaking countries but limited their search processes to mental health and public health settings. Our aim was to identify questionnaires used in German-speaking countries measuring the implementation of interventions in public health and health care settings in general and to assess their psychometric properties.
\end{abstract}

Methods: We searched five different bibliographic databases (from 1985 to August 2017) and used several other search strategies (e.g., reference lists, forward citation) to obtain our data. We assessed the instruments, which were identified in an independent dual review process, using 12 psychometric rating criteria. Finally, we mapped the instruments' scales and subscales in regard to the constructs of the Consolidated Framework for Implementation Research (CFIR) and the Implementation Outcome Framework (IOF).

Results: We identified 31 unique instruments available for the assessment of implementation science constructs. Hospitals and other health care settings were the ones most often investigated (23 instruments), while education and childcare settings, workplace settings, and community settings lacked published instruments. Internal consistency, face and content validity, usability, and structural validity were the aspects most often described. However, most studies did not report on test-retest reliability, known-groups validity, predictive criterion validity, or responsiveness. Overall, the majority of studies did not reveal high-quality instruments, especially regarding the psychometric criteria internal consistency, structural validity, and criterion validity. In addition, we seldom detected instruments operationalizing the CFIR domains intervention characteristics, outer setting, and process, and the IOF constructs adoption, fidelity, penetration, and sustainability.

Conclusions: Overall, a sustained and continuous effort is needed to improve the reliability and validity of existing instruments to new ones. Instruments applicable to the assessment of implementation constructs in public health and community settings are urgently needed.

Trial registration: The systematic review protocol was registered in PROSPERO on October 19, 2017, under the following number: CRD42017075208.

Keywords: Implementation variables, Psychometric properties, Test-theoretical criteria, Instrument, Questionnaires

\footnotetext{
* Correspondence: christina.kien@donau-uni.ac.at

${ }^{1}$ Department for Evidence-based Medicine and Clinical Epidemiology,

Danube-University Krems, Dr.-Karl-Dorrek Strasse 30, 3500, Krems a.d. Donau,

Austria

${ }^{2}$ Center for Public Health, Department of Social and Preventive Medicine,

Medical University Vienna, Kinderspitalgasse 15, 1090 Wien, Austria

Full list of author information is available at the end of the article
}

(c) The Author(s). 2018 Open Access This article is distributed under the terms of the Creative Commons Attribution 4.0 International License (http://creativecommons.org/licenses/by/4.0/), which permits unrestricted use, distribution, and reproduction in any medium, provided you give appropriate credit to the original author(s) and the source, provide a link to the Creative Commons license, and indicate if changes were made. The Creative Commons Public Domain Dedication waiver (http://creativecommons.org/publicdomain/zero/1.0/) applies to the data made available in this article, unless otherwise stated. 


\section{Background}

Clinical and health services research often takes up to 17 years or even fails altogether to translate into policy and practice $[1,2]$ resulting in an ineffective use of resources. Furthermore, in German-speaking countries, as in the rest of the world, there is a need to assess the implementation of evidence-based practices (EBP). Only if we can assess whether interventions are implemented properly will we know if they produce genuine public health effects [3]. In recent years, implementation science has increasingly relied on the use of theories, frameworks, and models to guide the implementation of evidencebased programs and to improve the planning of evaluation studies [4-6]. To support this use, overviews of theories $[4,7-10]$ as well as criteria and guidelines on how to select theories [5] have been published.

Despite this orientation towards theories, reliable and valid questionnaires to draw conclusions from evaluation studies would allow for greater advancements in implementation science and assist in closing the evidence-practice gap [11]. Knowledge can only be advanced when comparable, reliable, and valid questionnaires (i.e., instruments) are used to study implementation constructs (i.e., abstract phenomena that are not directly observable) and strategies [12]. Recent systematic reviews contributed to the field's development by revealing which theoretical domains and constructs associated with the adoption and implementation of programs could be assessed in a reliable and valid way [13-18]. Some limitations of previously conducted reviews [19] include the incomplete reporting of the instruments' psychometric properties (e.g., test-theoretical parameters, such as reliability and validity) and having an exclusive focus on their use in hospital and health care settings [20].

More recently, one initiative [21] and one systematic review [19] provided a more comprehensive perspective on the instruments' psychometric properties and covered a broad range of theoretical domains and constructs. The Society for Implementation Research Collaboration (SIRC) focused on the mental health care setting in their Instrument Review Project [21]. The review by Clinton-McHarg and colleagues [19] complemented this by concentrating on the public health care setting. Members of the SIRC Instrument Review Project team identified over 420 instruments [21] related to the Consolidated Framework for Implementation Research (CFIR) [22] and the review by Clinton-McHarg's group identified around 50 instruments related to CFIR's constructs. CFIR is considered to be a determinant framework. When developing CFIR, researchers analyzed the definitions and the terminology of several existing frameworks and theories and finally presented factors that act as barriers or enablers of the implementation process [4]. Overall, CFIR comprises 39 different constructs grouped into five different domains relating to intervention characteristics (e.g., evidence strength and quality, and complexity), outer setting (e.g., patient needs and resources), inner setting (e.g., implementation climate, network, and communication), characteristics of individuals (e.g., knowledge and beliefs about the intervention, self-efficacy), and process (e.g., planning, engaging). Furthermore, the SIRC Instrument Review Project team located more than 100 instruments [23] addressing domains of the Implementation Outcomes Framework (IOF) [24]. This framework covers eight different implementation outcomes. They are seen as revealing the effects of the implementation process and focus on the following aspects: acceptability, adoption, appropriateness, feasibility, fidelity, implementation cost, penetration, and sustainability of the intervention. Although both reviews applied comprehensive search strategies and assessment approaches, neither took into account the general hospital and health care settings besides mental health interventions [21], and Clinton-McHarg's group [19] did not include the domains of IOF as relevant outcomes.

Since implementation science is becoming more prevalent in German-speaking countries [25, 26], a systematic search for instruments that can be used with German-speaking populations is highly relevant. Furthermore, as most tools available for judging the influence of contextual factors or the implementation process on the effect of interventions have been developed in English-speaking countries, it remains hitherto unclear as to how many questionnaires might be available for this purpose in German. Unfortunately, the aforementioned reviews located only a single instrument developed and used in German. Since it would be vital for oversight bodies in German-speaking countries to possess tools so as to judge implementation outcomes, there is an urgent need to determine the number and quality of available instruments for this purpose.

To the best of our knowledge, no previous review has been conducted focusing on implementation constructs assessing instruments that are available in German and designed for use in public health and health care settings. The aims of this review-following a similar approach to those already conducted in this field [19, 21, 23]-were firstly to identify quantitative instruments assessing constructs described in CFIR [22] and IOF [24], which have been applied within a German-speaking population, and secondly to survey the psychometric properties of the identified instruments. CFIR and IOF were chosen because of their comprehensiveness and high usage rate in the evaluation of interventions [5].

\section{Methods}

We registered this review's protocol in PROSPERO (International Prospective Register of Systematic Reviews) under the registration number CRD42017075208. The 
design of the systematic review follows SIRC's Instrument Review Project [21] and Clinton-McHarg's group approach [19].

\section{Eligibility criteria}

We included publications if they (1) were published in peer-reviewed journals, (2) reported on quantitative instruments, such as questionnaires or surveys, which (3) were applied to assess the implementation of a specific psychosocial or health-related innovation or intervention, (4) assessed at least one of the 38 CFIR $^{1}$ [22] or one of the eight IOF [24] constructs, and (5) were developed for the use in public health (e.g., child care or community centers, schools, universities, workplaces, and prisons) and health care settings (e.g., hospitals, general practice, allied health facilities such as physiotherapy or dental practices, rehabilitation centers, psychiatric facilities). Furthermore, these instruments should have at least one aspect of reliability or validity assessed and should have been completed by German-speaking facilitators or participants of the interventions. We included the following psychometric properties in our review: internal consistency, construct validity, criterion validity, structural validity, responsiveness, face and content validity, norms, usability, and test-retest reliability.

\section{Data sources and search process}

We searched MEDLINE (via PubMed), PsycINFO (Ovid), PSYNDEX plus Literature and Audiovisual Media, PSYNDEXplus Tests and Education Resources Information Center (ERIC) from 1985 until August 2017. We assumed that no instrument would be published before 1985 as implementation science evolved later [21]. We selected these five databases, as they index relevant journals reporting on the evaluation of implementation of psychosocial or health-related interventions. The search strategy entailed the following elements and several variations of the search terms for a keyword search as well as for a search with Medical Subject Headings (MeSH) terms: (1) questionnaire, (2) psychometric properties, (3) intervention, and (4) implementation. We limited electronic searches to English and German as well as to human populations. Furthermore, we limited the search results to references with at least one author residing in a German-speaking country ("Affiliation" set to an institution in Austria, Germany, or Switzerland). We assumed that authors residing in German-speaking countries most likely would have tested their instruments on German-speaking population samples. We amended the search strategy developed in MEDLINE (via PubMed) to other databases. The detailed search strategy is presented in Additional file 1.

Additionally, we promoted our research project via a snowball sampling e-mail procedure to German-speaking experts in the field of implementation science and via an entry in the German-speaking Implementation Association's [26] newsletter, intending to identify further relevant publications. We also used several recent systematic reviews on this topic $[13,15,17,19,20,23,27]$ to check via forward citation tracking using Scopus if the instruments had been applied in German-speaking countries.

In a second step, we used already located instruments and continued the search process to detect further publications reporting on psychometric properties of these instruments. We searched the Scopus database by entering the name of the instrument in the search field and by using the forward citation tracking link of the source article.

\section{Study selection}

Two investigators independently reviewed abstracts and full-text articles according to a priori defined eligibility criteria and solved conflicts by discussion. All reviewers piloted the abstract and full-text review forms to test the applicability of inclusion and exclusion criteria. This process led to the refinement of the definitions of psychosocial and health-related interventions. The abstract review was carried out in AbstrackR [28]. We managed and saved all results of the abstract and full-text review including information on the reasons for exclusion in the full-text review in an Endnote database.

\section{Data extraction and rating process}

We piloted and improved the layout of the sheets and the rules for data extraction according to the feedback of the research team (e.g., how to deal with two studies reported in one paper). One reviewer extracted the pre-specified relevant data from eligible publications and a second reviewer checked the data for correctness. The reviewers solved discrepancies by consensus or by involving a third reviewer. We extracted data points relating to the development and assessment process of the instrument, to the description of the instrument, and to its psychometric characteristics.

\section{Development and assessment process}

This includes research setting, sample (gender and profession of participants answering the questionnaire), study characteristics (response rate), country where the instrument was developed, and characteristics of the intervention being assessed.

\section{Description of the instrument}

This embodies the name, abbreviation and aim of the instrument, number and names of subscales, and number of items.

\section{Psychometric properties}

This includes internal consistency (i.e., reliability), construct validity (convergent, discriminant, and known-groups), 
criterion validity (predictive and concurrent), structural validity (i.e., dimensionality), responsiveness, norms, and usability. Following Clinton-McHarg's group approach [19], we also included information on test-retest reliability, face, and content validity. Lewis and colleagues described evidence-based assessment (EBA) rating criteria that have undergone a thorough development process [21, 29] and were compiled in the Psychometric and Pragmatic Evidence Rating Scale (PAPERS). The scale includes six different rating levels with clearly defined cut-off values ranging from " $-1-$ poor", and " $0-$ no information available" to "4-excellent" for psychometric properties (Additional file 2). Two different investigators independently rated the psychometric properties for each individual study. Instruments that were assessed in more than one study received an overall rating applying the worst score counts approach (i.e., the worst rating achieved in different studies represented the final vote). We deviated from this practice in our assessment of the domain "norms". There, we used the best score counts approach as all interested researchers have access to the best available information.

After the assessment of the psychometric properties, one reviewer assigned the scale and subscales of the included instruments to 38 CFIR constructs and subscales [22] and eight IOF constructs [24]. A second reviewer checked this assignment. The mapping process focused on the description of the subscales and scales and not on the items.

\section{Analyses and reporting of the data}

We reported on the number of identified instruments and further used descriptive statistics (i.e., frequencies, mean, median, standard deviation, and range) to inform about the psychometric properties of the instruments and the results of the mapping process (assigning scales to the CFIR and IOF constructs). We used Microsoft Excel 2010 for calculating the descriptive statistics.

\section{Results}

First, we describe the results of the search process. Then, we present the identified instruments and their psychometric properties. Finally, we display the instruments' mapping against CFIR and IOF constructs.

\section{Results of the search process}

Our database search yielded 38 articles [30-67] reporting on the psychometric properties of 31 different instruments. The detailed flow of the literature selection process is depicted in Fig. 1. The majority of the instruments (23/31; $74 \%$ ) were developed for the use in hospital and health care settings [30-35, 38, 41-43, 47-51, 53-59, 63, 64, 67, 68]. Two instruments each were applied in the education $[36,52]$ and workplace settings $[39,45]$, and the psychometric properties of four instruments [37, 40, 44, $46,55,60-62,65,66]$ were assessed in more than one different setting (Table 1). Diverse interventions ranging from psychological and drug treatments to organizationwide implementation of quality improvement systems were evaluated using the identified instruments. Several questionnaires dealt with the assessment of web-based or technology-focused interventions. The number of subscales varied between one and 16 and the number of items per instrument ranged from two to $67[37,52]$. The majority of the studies were conducted in Germany $(n=21)$, followed by Austria $(n=11)$ and Switzerland $(n=4)$. The number of subscales varied between one and 16 and the number of items per instrument ranged from two to 67 . The development of 20 out of 31 identified instruments was based on other existing instruments available in English (e.g., translations of English original versions, see Additional file 3: File 5).

Overall, we identified only six instruments where the assessment process was based on different samples $[32,33,37,40,44,55-61,63,64,66]$, resulting in a more thorough assessment.

\section{Psychometric properties of the instruments}

The amount and the quality of information offered for each instrument varied considerably. On average, 4.9 out of 12 psychometric criteria were reported per instrument, ranging from three to nine criteria. Only ten instruments conveyed information on six or more different psychometric criteria [30-33, 37, 40,44, 48, 51, 55, 57-66]. All or most articles reported on usability (100\%) and internal consistency (97\%) of the scales (Table 2). In contrast, information on construct and criterion validity was rarely reported (6-16\%). No instrument reported on the psychometric property responsiveness.

The results for different settings can be found in Additional file 4.

The specific results for the included instruments are depicted in Fig. 2 for the hospital and health care settings and in Fig. 3 for the educational, workplace, and diverse settings. In the following sections, each psychometric property is described separately.

\section{Reliability-internal consistency}

This criterion refers to the extent that items on a scale or subscale can be correlated to each other due to their assessment of the same construct. The Cronbach's $\alpha$ coefficient is the most frequently used indicator [69]. Most instruments $(30 / 31$; 97\%) reported data on reliability of either the total scale or subscales. On average, the rating was $1.8(\mathrm{SD}=1.4)$, ranging from -1 to 4 . The median rating assigned to only those instruments, which provided information on that aspect, was 2.0 representing an "adequate" rating (Table 2). Nine instruments [31, 41, 44-47, 50, 52, 55, 


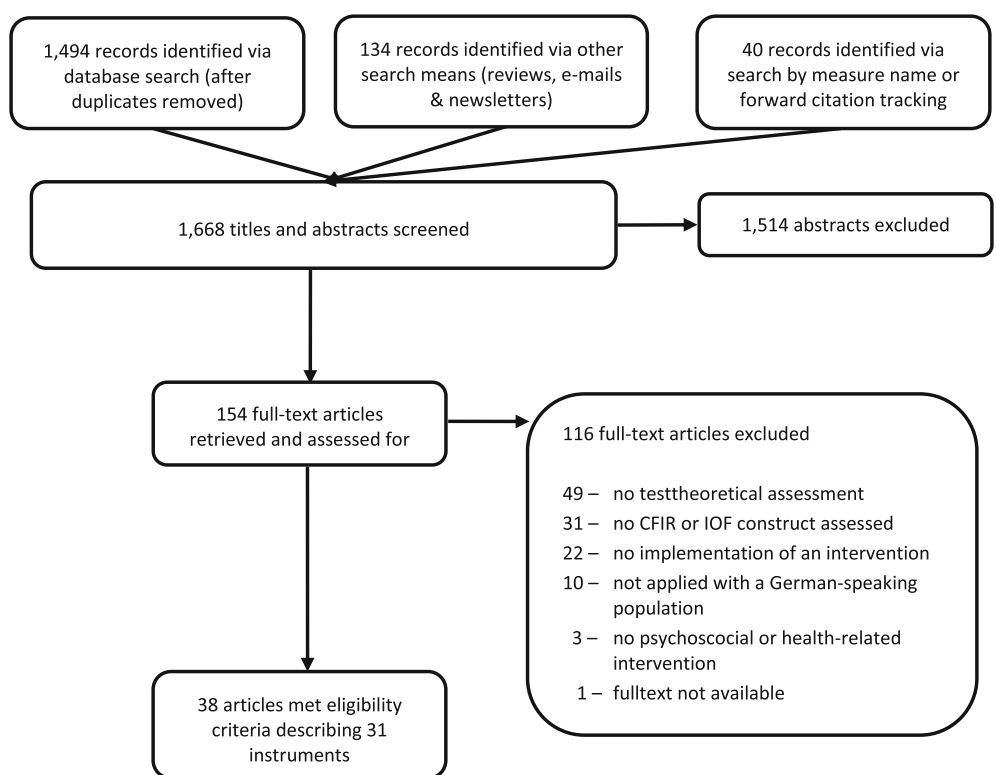

Fig. 1 PRISMA flow diagram of the study selection process

62, 67] showed at least adequate Cronbach's $\alpha$ values $(0.70<\alpha \leq 0.79)$. Only seven instruments received a good rating, $\alpha \geq 0.80[32,34,39,42,44,48,55,57,59$, $63,64,66]$ and three an excellent rating, $\alpha \geq 0.90$ [32, 33, $51,56,58]$. Cronbach's $\alpha$ values per instrument are depicted in Additional file 3: File 1.

\section{Construct validity-convergent, discriminant, and known- groups}

This term describes the extent that a group of items characterize the construct to be measured [70]. While convergent validity is seen as the accordance in empirical relatedness of theoretically allied constructs, discriminant validity is seen as the empirical discordance of theoretically unrelated constructs [70]. Known-groups validity seeks to determine whether groups with distinct features can be differentiated by their responses on a new instrument $[29,70]$.

Overall, only about a quarter of the instruments $(7 / 31$; $23 \%)$ informed on at least one aspect of construct validity (Table 2). However, if any authors offered information on those aspects, the median ratings showed good or excellent results (range, 3.5-4 points). Four instruments (CSQ-8, DTSQ-S, GSE, and SS-TC) disclosed information on convergent and discriminant validity [32, 44, 55, 57, 59, $62-64,66]$ and for one instrument each, information only on convergent validity [48] and discriminant validity [33, 58] was reported: the "Client Satisfaction QuestionnaireInternet" (CSQ-I) and the "Usefulness Scale for Patient Information Material" (USE), respectively. The median for instruments being tested for these validity aspects was 4.0 (Table 2). For two instruments [32, 48, 56], the authors reported on the assessment of known-groups validity (Table 2). The "Diabetes Treatment Questionnaire Change" (DTQ-C) $[32,56]$ received a rating of " $4-$ excellent" (i.e., two or more statistically significant differences between groups detected and hypotheses tested) and the USE [48] received a rating of "3-good" (i.e., one expected difference was shown between groups). Detailed information regarding construct validity can be found in Additional file 3: File 2.

\section{Criterion validity-predictive and concurrent}

This criterion refers to the extent to which a new instrument is correlated with a "gold standard" (i.e., measuring a distinct outcome). If an instrument is additionally administered at some point in the future, it refers to predictive validity. If it is administered at the same time, the validity aspect is called concurrent validity [69]. The CSQ-8 [59, 63, 64] and the SAMS-P/SAMS-S [51] reported data on both aspects, predictive and concurrent validity. Additionally, authors provided data on concurrent validity for eight other questionnaires [30,31, 35, 37, 40, 41, 43-45, 60-62]. The median rating was 1.0 for predictive validity and 2.0 for concurrent validity (Table 2). The CSQ-8 [59, 63, 64] and the SAMS-P/SAMS-S [51] received only a rating of " $1-$ minimal/emerging validity" for the predictive validity (i.e., Pearson's $r$ reached only a value between 0.10-0.29). Only two out of ten instruments including the CSQ-8 $[59,63,64]$ and the 'Patients' Experiences Across Health Care Sectors" (PEACS) [35] verified a "3-good" concurrent validity (i.e., $0.50<$ Pearson's $r \leq 0.69$; see Additional file 3: File 3). 







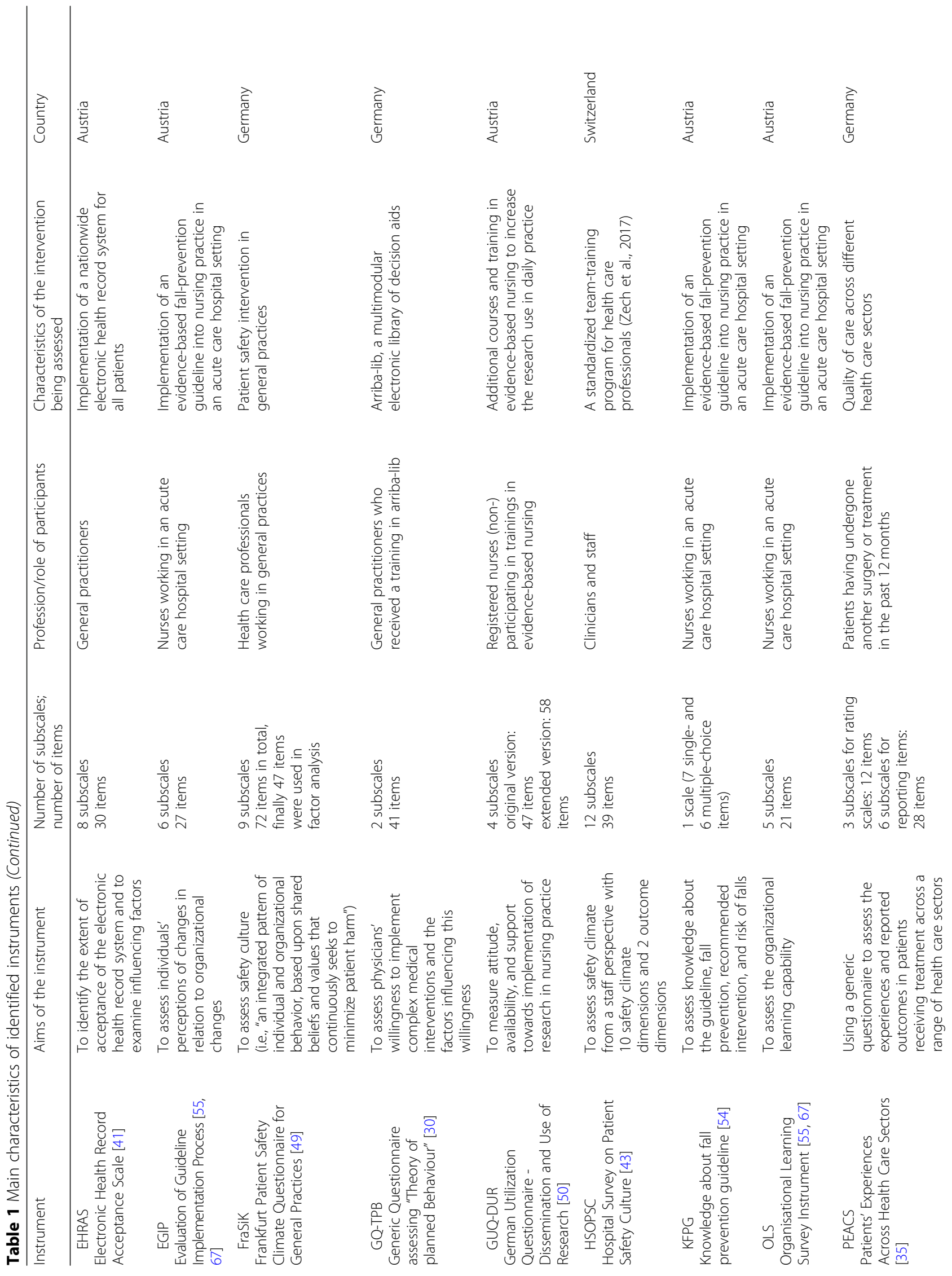




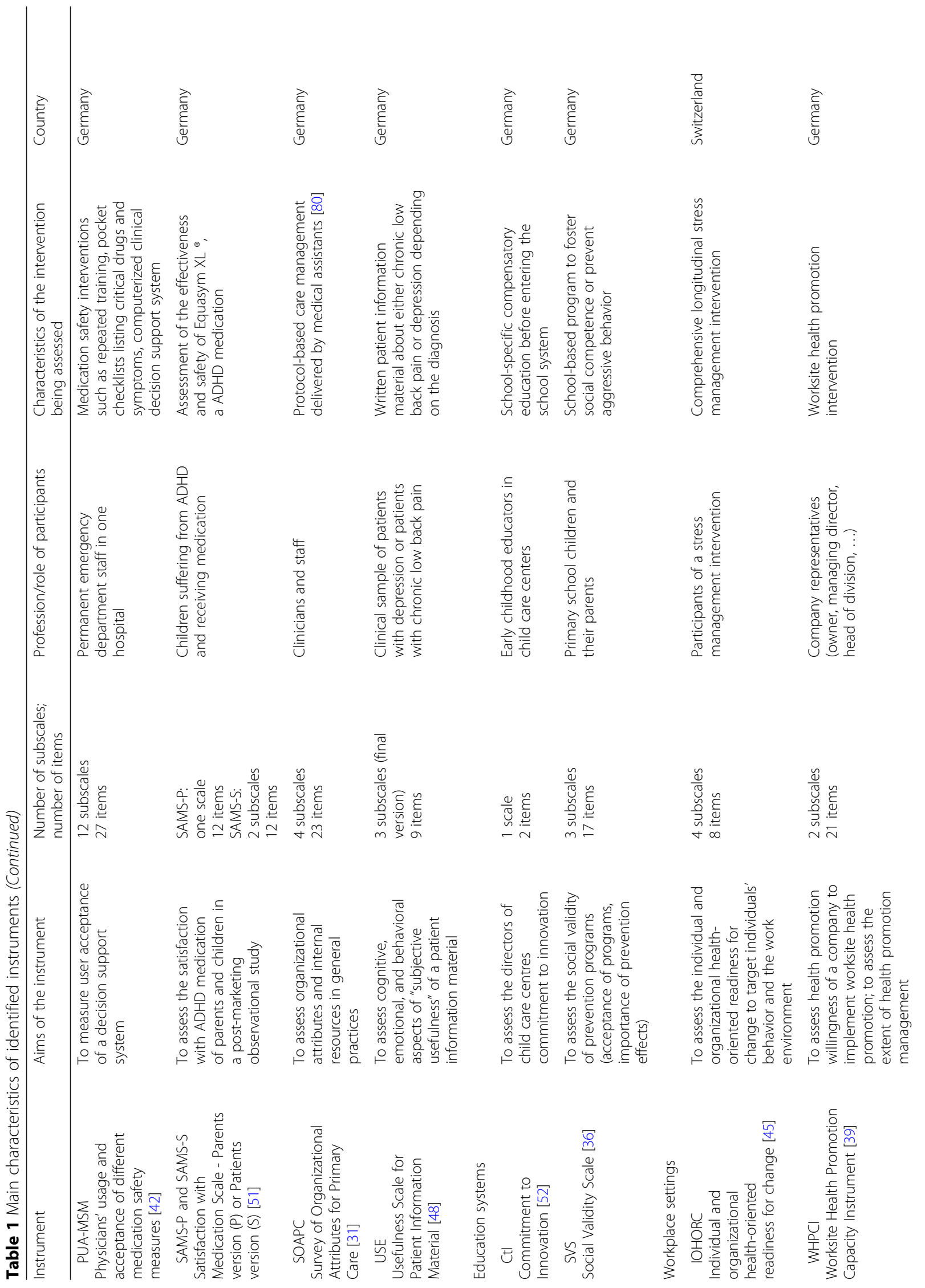




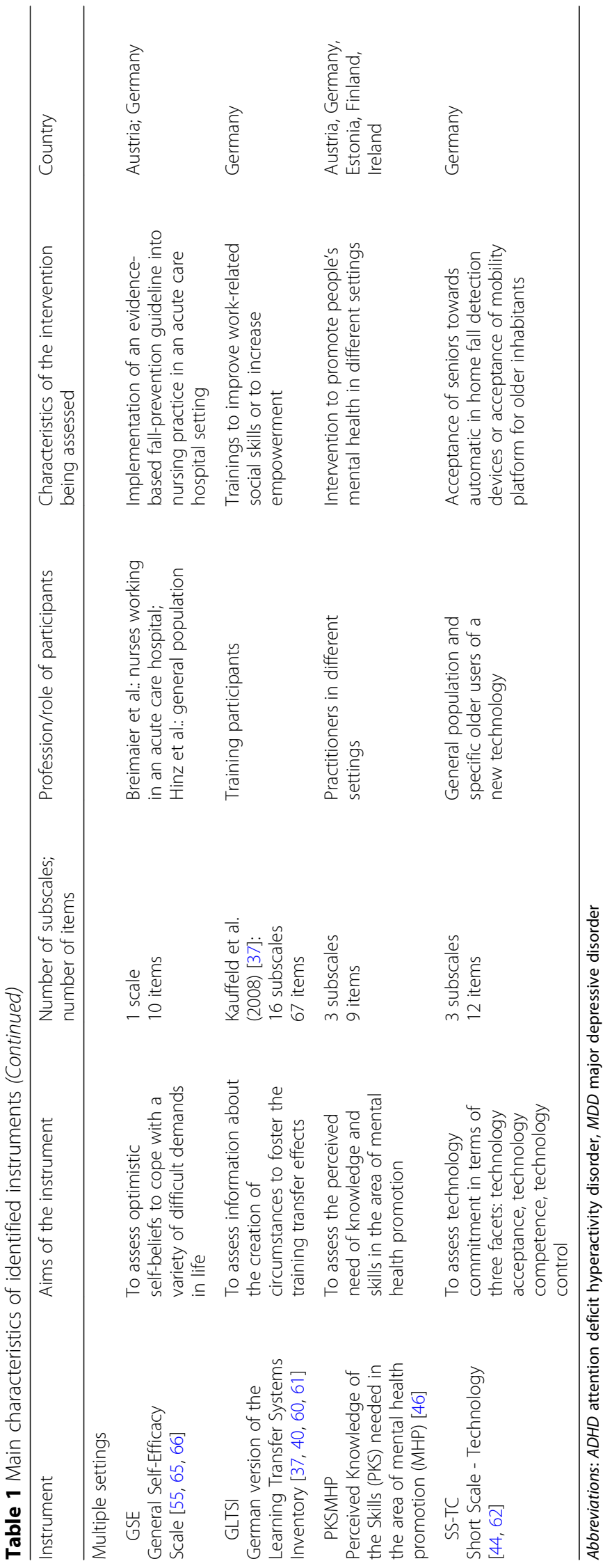


Table 2 Overview of psychometric properties of the instruments

\begin{tabular}{llllllll}
\hline Psychometric properties & $N$ & $\%$ & $M$ & SD & Md & min & $\max$ \\
\hline Internal consistency & 30 & 97 & 1.8 & 1.4 & 2.0 & -1 & 4 \\
Convergent validity & 5 & 16 & 0.6 & 1.4 & 4.0 & 0 & 4 \\
Discriminant validity & 5 & 16 & 0.6 & 1.4 & 4.0 & 0 & 4 \\
Known-groups validity & 2 & 6 & 0.2 & 0.9 & 3.5 & 0 & 4 \\
Predictive validity & 2 & 6 & 0.1 & 0.2 & 1.0 & 0 & 1 \\
Concurrent validity & 10 & 32 & 0.5 & 1.0 & 2.0 & -1 & 3 \\
Structural validity & 21 & 68 & 1.5 & 1.8 & 2.0 & -1 & 4 \\
Responsiveness & 0 & 0 & 0.0 & 0.0 & 0.0 & 0 & 0 \\
Norms & 14 & 45 & 1.3 & 1.6 & 3.0 & 0 & 4 \\
Usability & 31 & 100 & 3.2 & 0.6 & 3.0 & 2 & 4 \\
Test-retest reliability & 3 & 10 & 0.1 & 0.3 & 1.0 & 0 & 1 \\
Face and content validity & 29 & 94 & 0.9 & 0.2 & 1.0 & 0 & 1 \\
\hline
\end{tabular}

Abbreviations: $M$ mean over all ratings, max maximum, $M d$ median rating considering only those instruments which provided information on that aspect, min minimum, $n$ number of instruments with a rating of $-1,1,2,3$ or $4 ; \%$, percentage of instruments with a rating of $-1,1,2,3$ or $4, S D$ standard deviation over all ratings

Explanation: This table displays the aggregated rating information for each psychometric property based on 31 identified instruments. Rating ranges from - 1 "poor", 0 "no information", 1 "minimal emerging", 2 "adequate", 3 "good", 4 "excellent" for all the psychometric properties except test-retest reliability, and face and content validity where the rating was 0 "no information provided" and 1 "information provided"

\section{Dimensionality-structural validity}

This term is defined as the extent to which an instrument reveals the internal structure of its components as expected or theoretically hypothesized [69]. A prominent way to assess structural validity is via factor analysis. Authors of two thirds of the instruments $(21 / 31,68 \%)$ revealed information on aspects of structural validity [30-40, 43-46, 48, $49,51,53,55-66]$. Overall, the median rating for structural validity was 2.0 , showing a wide variety, mirrored in the ratings, ranging from -1 to 4 (Table 2). For example, the explained variance of the factor analyses stretched between $35 \%[38]$ and $75 \%[32,56]$. Six instruments including the CSQ-8 [59, 63, 64], the DTSQ-S [32, 57], the SAMS-P/ SAMS-S [51], the "Survey of Organizational Attributes for Primary Care" (SOAPC) [31], the "Worksite Health Promotion Capacity Instrument" (WHPCI) [39], and the GSE $[55,65,66]$ reached an excellent structural validity rating, as the explained variance was $>50 \%$ and the sample size was sufficiently large. The best rating (see Additional file 3: File 1) for the assessment of confirmatory factor analysis was "3-good," which was awarded to two instruments: the "Social Validity Scale" (SVS) [36] and the "Individual and organizational health-oriented readiness for change questionnaire" (IOHORC) [45].



Fig. 2 PAPERS rating criteria of instruments used in the hospital and health care setting 


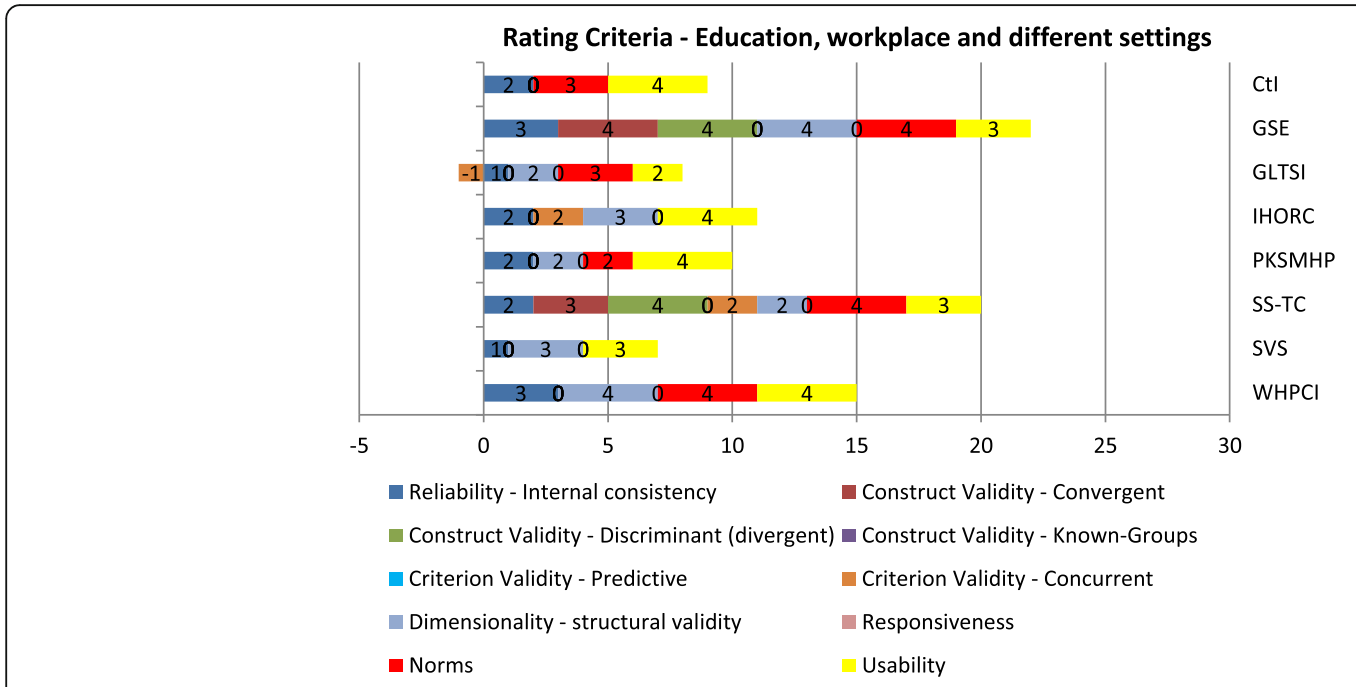

Fig. 3 PAPERS rating criteria of instruments used in the education setting, workplace setting, and different settings

\section{Norms}

Norms in terms of central tendency and distribution of the total score [29] were available for about half $(14 / 31$, $45 \%)$ of the instruments [31, 33, 34, 37, 39-41, 44, 46, $50-52,54,55,58-66]$. The median for the rating of this dimension was "3-good," ranging from 0 to 4 (Table 2). Age- and gender-specific norms (see Additional file 3: File 4) were only available for the GSE $[65,66]$.

\section{Usability}

This is a pragmatic criterion that refers to the ease of use in terms of the necessary number of items to measure a construct. This criterion was not included in the PAPERS criteria [29], but in the first rating scale version designed by Lewis and colleagues [21]. All instruments revealed information on usability. Ten instruments had fewer than ten items [32, 33, 39, 45-48, 52, 56-59, 63, 64], receiving a "4-excellent" rating, and 18 instruments had greater than ten but fewer than 50 items, receiving a "3-good" rating. The median rating was 3.0, ranging from 0 to 4 (Table 2). Clinton-McHarg and colleagues [19] also considered the number of missing items observed following instrument administration. Overall, eight instruments reported on the maximum value or range of missing values $[30,31,35,39,41,45,46,49]$. The reported maximum percentage of missing values was $13.2 \%$ for a specific item in the "Perceived Knowledge of the Skills needed in the area of Mental Health Promotion scale" (PKSMHP) [46]. Detailed information can be found in Additional file 3: File 4.

\section{Test-retest reliability}

This criteria is defined as the stability of the instrument over time [70]. This aspect was not included in the PAPERS criteria [29]. Only three instruments reported on test-retest reliability: the "Generic Questionnaire assessing 'Theory of planned Behaviour"' (GQ-TPB) [30], the PEACS [35], and the SAMS-P/SAMS-S [51]. Only the assessment study of GQ-TPB [30] applied the appropriate administration period of 2 to 14 days while the others $[35,51]$ relied on a longer administration period ( 3 to 10 weeks). None of the instruments received adequate test-retest reliability $(r>0.70)$ for all of the subscales. The test-retest coefficients ranged between 0.54 and 0.86 (see Additional file 3: File 3).

\section{Face and content validity}

Face validity refers to the extent researchers and those who complete an instrument agree that the instrument measures what it purports to measure [70]. Content validity refers to the instrument's development process and considers selection of items, theory relatedness, and formal assessment of the instrument's content [19]. Neither aspect was included in the PAPERS criteria [29]. Most of the instruments (94\%) provided background on their instrument's development process. Authors used theoretical knowledge in the development process of 19 instruments. To improve face and content validity, researchers of 15 instruments applied diverse methods such as expert ratings of the draft version, Delphi groups, pre-testing of instruments with the intended population, and cognitive pre-tests (see Additional file 3: File 5).

\section{Responsiveness}

This refers to the ability of an instrument to detect change over time $[29,71]$. No instrument provided data on this dimension.

\section{Mapping against CFIR and IOF constructs}

A total of 19 instruments included at least one of the 38 CFIR constructs (see Additional file 5). On average, each 
instrument assessed two constructs, ranging from one to seven constructs. The "German version of the Learning Transfer Systems Inventory" (GLTSI) [37, 40, 60, 61] measured seven constructs. Overall, the different constructs were investigated rather unevenly. Two CFIR constructs, networks \& communications [31, 37, 43, 49, 50, 55, 67] and individual's knowledge and beliefs about the intervention [30,37, 41, 44, 45, 54] were assessed six times, and the domain leadership engagement $[37,43,49,52$, $55,67]$ was operationalized five times. However, 22 constructs of the CFIR framework were not covered by instruments in German at all. The majority of those belonged to the CFIR domains intervention characteristics, outer setting, and process. The domain inner setting, however, was investigated intensively: 13 instruments covered the 14 CFIR constructs of that domain a total of 25 times.

Altogether, 17 instruments enabled users to assess at least one of IOF's eight constructs. On average, one instrument enabled the testing of 1.4 IOF constructs. Overall, it ranged from one to three constructs [37, 40, 60, 61]. These instruments were the "Acceptance of Mobile Mental Health Treatment Applications scale" (AMMHTA) [53] and the "Attitudes towards Guidelines Scale" (AGS) [54]. The most frequently $(n=16)$ operationalized domain of IOF was acceptability, followed by feasibility $(n=4)$, appropriateness $(n=3)$, and cost $(n=1)$. No instrument covered the domains adoption, fidelity, penetration, and sustainability.

\section{Discussion}

Currently, there is a lack of instruments available for assessing implementation processes in German-speaking countries. Several initiatives and reviews $[19,21]$ have recently been conducted to locate questionnaires that assessed contextual factors influencing implementation processes and outcomes. Nevertheless, only one questionnaire was identified that had been adapted for use in the German language. Hence, we conducted a systematic review to detect instruments used for measuring implementation constructs specifically in the German language. Overall, we identified 38 articles reporting on the psychometric properties of 31 instruments. While we could identify 23 different instruments for the hospital and health care setting, comparably fewer published instruments could be identified for other settings (e.g., workplace, community, education, and childcare settings). On average, each instrument provided information on 4.9 out of 12 psychometric criteria, ranging from three to nine. Generally, most articles provided information on the internal consistency (97\%) but, authors rarely reported on construct validity (23\%). The fact that validity aspects were not reported was reflected by other reviews in this area [19-21, 23]. The missing information on validity is significant as it is unclear whether or not the instruments are actually measuring what they intend to measure and if the conclusions based on this research are valid and meaningful.

Furthermore, the quality of information described for reliability was only "2-adequate". Overall, these results show that the majority of the currently applied instruments require further refinement, more extensive item development, and retesting of scales. Without well-developed instruments, researchers will continue to use self-developed instruments, which will impair the ability of the implementation science community in German-speaking countries to further test theories and advance the field's knowledge. When researchers use existing instruments with low validity and reliability, they should be aware that results have to be interpreted with caution and that they should use multiple sources for assessing implementation variables [72].

Some of the instruments showed reliable results, especially the ones assessing the IOF construct acceptability, such as the "Client Satisfaction Questionnaire" (CSQ-8) [59, 63, 64] and the "Diabetes Treatment Satisfaction Questionnaire Status" (DTSQ-S) [32, 57]. The CSQ-8 received 26 out of 40 possible points and the DTSQ-S attained 19 points (Figs. 2 and 3): Two instruments, the "General Self-Efficacy Scale" (GSE) $[55,65,66]$ and the "Short Scale - Technology Commitment" (SS-TC) [44, 62] used in settings other than in hospitals and health care facilities also showed a profound assessment of six different psychometric criteria, achieving 22 and 20 points, respectively.

Overall, the identified instruments contributed very unevenly to the 38 CFIR and eight IOF constructs. The questionnaires exposed here covered 20 out of 46 constructs of the aforementioned frameworks. Specifically, a serious shortage in instruments could be attributed to the CFIR domains intervention characteristics, outer setting, and process as well as the IOF constructs adoption, fidelity, penetration, and sustainability. While a review of instruments in the field of mental health [21] found a similar majority of instruments assessing acceptability, the high number of identified instruments in their review for the construct adoption in comparison to our review was surprising. This may be partly due to the different coding processes of the reviewers. Despite the high number of instruments assessing acceptability and appropriateness, instruments operationalizing these constructs in the public health and community settings or in a generic way were scarce. To foster the knowledge generation in that area, these instruments need to be developed. Furthermore, the CFIR subdomains intervention characteristics, outer setting, and process require future attention regarding the development process of instruments [19, 21]. Both reviews by the groups of Lewis and Clinton-McHarg [19, 21] mirrored the findings of the most frequently assessed domains being inner setting and characteristics of individuals. 
In general, the overlap of identified instruments between our study and the aforementioned systematic reviews [19, 21] was rather minimal. The missing congruency might be attributed to the different foci and inclusion criteria of the reviews: Lewis and colleagues [21] focused on mental health interventions, while we did not include instruments assessing the day-to-day psychotherapeutic treatment. While Clinton-McHarg's group [19] included only studies conducted in the public health sector assessing CFIR but not IOF constructs, our review included the general hospital and health care settings as well, where most instruments had been applied. Another difference between the previously conducted reviews and our work was that the former excluded studies not published in English [19, 21], and therefore, those instruments published in German were not included [52, 62, 63]. Clinton-McHarg et al. [19] showed that the majority of the instruments (38 out of 51) were developed in the USA, Canada, and other English-speaking countries, thereby revealing the prominent position of the English-speaking implementation science community. This has been reflected by our result that the development of 20 out of 31 identified instruments was based on other existing instruments available in English (e.g., translations of English original versions). And while both instruments were captured by the different searches and identification processes, some instruments in German [42, 50] were adaptations of the original versions in English [73, 74] and, therefore, were not explicitly listed in the aforementioned reviews and vice versa.

\section{Limitations}

Despite a thoroughly developed and tested bibliographic search strategy, some relevant publications may have been missed. To combat potential drawbacks of our strategy, we extended our searches to include citation forward techniques and approached experts for suggestions of eligible articles [75]. Nevertheless, it is important to mention that we only used the defined source article by the SIRC review team for forward citation search, although often more than one reference was listed. If authors residing in German-speaking countries relied on another publication, we would not have been able to identify it. Similar to the approach by Clinton-McHarg and her group [19], we did not rely on gray literature searches, assuming that authors taking the thorough effort of developing or translating a well-designed instrument $[69,70,76]$ would publish it in indexed journals. Furthermore, as we were interested in instruments which have already been used for the evaluation of an intervention, we did not include studies that covered CFIR constructs that had not been used in such an assessment process [77-79]. As mentioned above, a further limitation of the review was that the alignment of the identified scales and subscales to the CFIR and IOF constructs was done on scale but not item level. Some misclassifications may have happened as no clear and non-overlapping definitions of constructs are currently available [24].

Nevertheless, the present work provided an overview including an evaluation of the instruments' psychometric properties of available German instruments used for assessing implementation constructs. This readily available information can guide future research efforts in this area. For existing instruments, it seems to be necessary to improve the internal consistency of the scales and to promote research on construct and criterion validity. Furthermore, the mapping process onto the CFIR and IOF constructs revealed that instruments assessing the CFIR domains intervention characteristics, outer setting, and process and the IOF domains adoption, fidelity, penetration, and sustainability are missing. In addition, one generic questionnaire measuring the most relevant IOF constructs including acceptability, appropriateness, and feasibility would advance the field.

\section{Conclusions}

Some instruments (e.g., CSQ-8, DTSQ-S, GSE, and SS-TC) present a good starting point for assessing relevant CFIR and IOF constructs in the German language. Nevertheless, a continuous effort is needed for the improvement of existing instruments regarding the reliability and construct validity in particular, but also for the development of relevant missing instruments. This is especially significant for instruments in the public health and community settings. We encourage pooling the efforts in the German language implementation science community to prioritize which instruments should be developed or translated. In this way, German-speaking implementation researchers can foster a reliable and valid operationalization of implementation frameworks in multiple contexts while promoting an economically sensible use of research resources.

\section{Endnotes}

${ }^{1}$ We did not include the "other personal attributes" construct of the CFIR domain "characteristics of individuals" as no clear definition was available to guide a selection process.

\section{Additional files}

\footnotetext{
Additional file 1: Documentation search strategy. (DOCX $31 \mathrm{~kb}$ )

Additional file 2: Description rating criteria. (DOCX $43 \mathrm{~kb}$ )

Additional file 3: 1 . Details psychometric criteria-reliability and structural validity. 2 Details psychometric criteria-construct validity. 3 Details psychometric criteria—criterion validity, test-retest reliability. 4. Details psychometric criteria—norms, usability. 5. Details psychometric criteria-face and content validity, responsiveness. (ZIP $158 \mathrm{~kb}$ )
} 
Additional file 4: Overview of instrument's psychometric properties used in settings. (DOCX $27 \mathrm{~kb}$ )

Additional file 5: Mapping CFIR and IOF constructs. (DOCX 66 kb)

\section{Abbreviations}

ADHD: Attention deficit hyperactivity disorder; CFIR: Consolidated Framework for Implementation Research; EBP: Evidence-based practices;

IOF: Implementation Outcomes Framework; M: Mean; Max: Maximum; Md: Median; MDD: Major depressive disorder; Min: Minimum; n: Number; PRISMA: Preferred Reporting Items for Systematic Reviews and Meta-Analyses; SD: Standard deviation; SIRC: Society for Implementation Research Collaboration

\section{Acknowledgements}

The authors wish to acknowledge the feedback of the information specialist Irma Klerings to the search protocol. We are grateful to Aner Gurvitz for his valuable comments on an earlier draft of this manuscript.

\section{Funding}

This research did not receive any specific grant from funding agencies in the public, commercial, or not-for-profit sectors.

\section{Availability of data and materials}

The datasets used and/or analyzed during the current study are available from the corresponding author on reasonable request.

\section{Authors' contributions}

CK drafted the research protocol and MTS, RS, and GG provided substantial contributions to the protocol. CK, MTS, and MS contributed to the abstract and full-text screening, data extraction, and evidence-based assessment of the instruments. CK wrote the first draft of the manuscript. All authors revised it critically for important intellectual content, and all approved the final manuscript.

\section{Ethics approval and consent to participate}

Not applicable.

\section{Consent for publication}

Not applicable.

\section{Competing interests}

The authors declare that they have no competing interests.

\section{Publisher's Note}

Springer Nature remains neutral with regard to jurisdictional claims in published maps and institutional affiliations.

\section{Author details}

'Department for Evidence-based Medicine and Clinical Epidemiology, Danube-University Krems, Dr.-Karl-Dorrek Strasse 30, 3500, Krems a.d. Donau, Austria. ${ }^{2}$ Center for Public Health, Department of Social and Preventive Medicine, Medical University Vienna, Kinderspitalgasse 15, 1090 Wien, Austria. ${ }^{3}$ Department of Applied Psychology: Work, Education, Economy, Faculty of Psychology, University of Vienna, Universitaetsstrasse 7, 1010 Vienna, Austria. ${ }^{4}$ Department of Maternal and Child Health, Gillings School of Global Public Health, University of North Carolina at Chapel Hill, CB \#7445 Rosenau, Chapel Hill, NC 27599-7445, USA. ${ }^{5}$ RTI International-University of North Carolina at Chapel Hill Evidence-based Practice Center, Chapel Hill 27599-7445, NC, USA.

Received: 31 May 2018 Accepted: 12 November 2018 Published online: 12 December 2018

\section{References}

1. Morris ZS, Wooding S, Grant J. The answer is 17 years, what is the question: understanding time lags in translational research. J R Soc Med. 2011;104(12): 510-20. https://doi.org/10.1258/jrsm.2011.110180.

2. Grimshaw JM, Eccles MP, Lavis JN, Hill SJ, Squires JE. Knowledge translation of research findings. Implement Sci. 2012;7(1):50. https://doi.org/10.1186/ 1748-5908-7-50.
3. Bauer MS, Damschroder L, Hagedorn H, Smith J, Kilbourne AM. An introduction to implementation science for the non-specialist. BMC Psychol. 2015;3:32. https://doi.org/10.1186/s40359-015-0089-9.

4. Nilsen P. Making sense of implementation theories, models and frameworks. Implement Sci. 2015;10:53. https://doi.org/10.1186/s13012-015-0242-0.

5. Birken SA, Powell BJ, Shea CM, Haines ER, Alexis Kirk M, Leeman J, et al. Criteria for selecting implementation science theories and frameworks: results from an international survey. Implement Sci. 2017;12(1):124. https:// doi.org/10.1186/s13012-017-0656-y.

6. Liang L, Bernhardsson S, Vernooij RW, Armstrong MJ, Bussieres A, Brouwers $M C$, et al. Use of theory to plan or evaluate guideline implementation among physicians: a scoping review. Implement Sci. 2017;12(1):26. https:// doi.org/10.1186/s13012-017-0557-0.

7. Tabak RG, Khoong EC, Chambers DA, Brownson RC. Bridging research and practice: models for dissemination and implementation research. Am J Prev Med. 2012;43(3):337-50. https://doi.org/10.1016/j.amepre.2012.05.024.

8. Wisdom JP, Chor KH, Hoagwood KE, Horwitz SM. Innovation adoption: a review of theories and constructs. Admin Pol Ment Health. 2014. https://doi. org/10.1007/s10488-013-0486-4.

9. Bergeron K, Abdi S, DeCorby K, Mensah G, Rempel B, Manson H. Theories, models and frameworks used in capacity building interventions relevant to public health: a systematic review. BMC Public Health. 2017;17(1):914. https://doi.org/10.1186/s12889-017-4919-y.

10. Moullin JC, Sabater-Hernandez D, Fernandez-Llimos F, Benrimoj SI. A systematic review of implementation frameworks of innovations in healthcare and resulting generic implementation framework. Health Res Policy Syst. 2015;13:16. https://doi.org/10.1186/s12961-015-0005-z.

11. Martinez RG, Lewis CC, Weiner BJ. Instrumentation issues in implementation science. Implement Sci. 2014. https://doi.org/10.1186/s13012-014-0118-8.

12. May C. Towards a general theory of implementation. Implement Sci. 2013. https://doi.org/10.1186/1748-5908-8-18.

13. Chor KH, Wisdom JP, Olin SCS, Hoagwood KE, Horwitz SM. Measures for predictors of innovation adoption. Admin Pol Ment Health. 2015. https:// doi.org/10.1007/s10488-014-0551-7.

14. Walters SJ, Stern C, Robertson-Malt S. The measurement of collaboration within healthcare settings: a systematic review of measurement properties of instruments. JBI Database System Rev Implement Rep. 2016;14(4):138-97. https://doi.org/10.11124/jbisrir-2016-2159.

15. Ramelow D, Currie D, Felder-Puig R. The assessment of school climate: review and appraisal of published student-report measures. J Psychoeduc Assess. 2015. https://doi.org/10.1177/0734282915584852.

16. Weiner BJ, Amick H, Lee SYD. Conceptualization and measurement of organizational readiness for change: a review of the literature in health services research and other fields. Med Care Res Rev. 2008. https://doi.org/ 10.1177/1077558708317802.

17. Allen JD, Towne SD Jr, Maxwell AE, DiMartino L, Leyva B, Bowen DJ, et al. Measures of organizational characteristics associated with adoption and/or implementation of innovations: a systematic review. BMC Health Serv Res. 2017;17(1):591. https://doi.org/10.1186/s12913-017-2459-x.

18. Emmons KM, Weiner B, Fernandez ME, Tu SP. Systems antecedents for dissemination and implementation: a review and analysis of measures. Health Educ Behav. 2012. https://doi.org/10.1177/1090198111409748.

19. Clinton-McHarg T, Yoong SL, Tzelepis F, Regan T, Fielding A, Skelton E, et al. Psychometric properties of implementation measures for public health and community settings and mapping of constructs against the Consolidated Framework for Implementation Research: a systematic review. Implement Sci. 2016;11(1):148. https://doi.org/10.1186/s13012-016-0512-5.

20. Chaudoir SR, Dugan AG, Barr CH. Measuring factors affecting implementation of health innovations: a systematic review of structural, organizational, provider, patient, and innovation level measures. Implement Sci. 2013;8:22. https://doi.org/10.1186/1748-5908-8-22.

21. Lewis CC, Stanick CF, Martinez RG, Weiner BJ, Kim M, Barwick M, et al. The Society for Implementation Research Collaboration Instrument Review Project: a methodology to promote rigorous evaluation. Implement Sci. 2015;10:2. https://doi.org/10.1186/s13012-014-0193-x.

22. Damschroder LJ, Aron DC, Keith RE, Kirsh SR, Alexander JA, Lowery JC. Fostering implementation of health services research findings into practice: a consolidated framework for advancing implementation science. Implement Sci. 2009;4:50. https://doi.org/10.1186/1748-5908-4-50.

23. Lewis CC, Fischer S, Weiner BJ, Stanick C, Kim M, Martinez RG. Outcomes for implementation science: an enhanced systematic review of instruments 
using evidence-based rating criteria. Implement Sci. 2015;10:155. https://doi. org/10.1186/s13012-015-0342-X

24. Proctor $E$, Silmere $H$, Raghavan $R$, Hovmand $P$, Aarons $G$, Bunger A, et al. Outcomes for implementation research: conceptual distinctions, measurement challenges, and research agenda. Admin Pol Ment Health 2011;38(2):65-76. https://doi.org/10.1007/s10488-010-0319-7.

25. Ullrich C, Mahler C, Forstner J, Szecsenyi J, Wensing M. Teaching implementation science in a new Master of Science Program in Germany: a survey of stakeholder expectations. Implement Sci. 2017;12(1):55. https://doi. org/10.1186/s13012-017-0583-y.

26. German Speaking Implementation Association (GSIA). German Speaking Implementation Association. 2018. Available from: http://www.implementation. eu/networks/german-speaking-implementation-association-gsia. Accessed: 18 Feb 2018.

27. Manser T, Brosterhaus M, Hammer A. You can't improve what you don't measure: safety climate measures available in the German-speaking countries to support safety culture development in healthcare. Z Evid Fortbild Qual Gesundhwes. 2016;114:58-71. https://doi.org/10.1016/j.zefq. 2016.07.003.

28. Brown University. AbstrackR beta-version 2017. Available from: http:// abstrackr.cebm.brown.edu/account/login. Accessed: 18 Feb 2018.

29. Lewis CC, Mettert K, Dorsey C, Martinez RG, Weiner BJ, Nolen E, et al. An updated protocol for a systematic review of implementation-related measures. Systematic Reviews. 2018. https://doi.org/10.1186/s13643-018-0728-3.

30. Kramer L, Hirsch O, Becker A, Donner-Banzhoff N. Development and validation of a generic questionnaire for the implementation of complex medical interventions. Ger Med Sci. 2014. https://doi.org/10.3205/000193.

31. Ose D, Freund T, Kunz CU, Szecsenyi J, Natanzon I, Trieschmann J, et al. Measuring organizational attributes in primary care: a validation study in Germany. J Eval Clin Pract. 2010. https://doi.org/10.1111/j.1365-2753.2009.01330.x.

32. Howorka K, Pumprla J, Schlusche C, Wagner-Nosiska D, Schabmann A, Bradley C. Dealing with ceiling baseline treatment satisfaction level in patients with diabetes under flexible, functional insulin treatment: assessment of improvements in treatment satisfaction with a new insulin analogue. Qual Life Res. 2000. https://doi.org/10.1023/A:1008921419108.

33. Bos $L$, Lehr D, Reis D, Vis C, Riper $H$, Berking M, et al. Reliability and validity of assessing user satisfaction with web-based health interventions. J Med Internet Res. 2016;18(8):e234. https://doi.org/10.2196/jmir.5952.

34. Schroder J, Berger T, Meyer B, Lutz W, Hautzinger M, Spath C, et al. Attitudes towards internet interventions among psychotherapists and individuals with mild to moderate depression symptoms. Cognitive Ther Res. 2017. https://doi.org/10.1007/s10608-017-9850-0.

35. Noest S, Ludt S, Klingenberg A, Glassen K, Heiss F, Ose D, et al. Involving patients in detecting quality gaps in a fragmented healthcare system: development of a questionnaire for Patients' Experiences Across Health Care Sectors (PEACS). Int J Qual Health C. 2014;26(3):240-9. https://doi.org/ 10.1093/intahc/mzu044.

36. Grumm M, Hein S, Fingerle M. Measuring acceptance of prevention programmes in children. Eur J Dev Psychol. 2013. https://doi.org/10.1080/ 17405629.2012.707311.

37. Kauffeld S, Bates R, Holton EF, Muller AC. The German version of the Learning Transfer Systems Inventory (GLTSI): psychometric validation. Z Personalpsychol. 2008. https://doi.org/10.1026/1617-6391.7.2.50.

38. Schroeder J, Sautier L, Kriston L, Berger T, Meyer B, Spaeth C, et al. Development of a questionnaire measuring attitudes towards psychological online interventions. J Affect Disord. 2015. https://doi.org/10.1016/j.jad.2015.08.044.

39. Jung J, Nitzsche A, Neumann M, Wirtz M, Kowalski C, Wasem J, et al. The Worksite Health Promotion Capacity Instrument (WHPCI): development, validation and approaches for determining companies' levels of health promotion capacity. BMC Public Health. 2010;10:550. https://doi.org/10. 1186/1471-2458-10-550.

40. Bates R, Kauffeld S, Holton EF. Examining the factor structure and predictive ability of the German-version of the Learning Transfer Systems Inventory. J Eur Ind Train. 2007. https://doi.org/10.1108/03090590710739278.

41. Steininger K, Stiglbauer B. EHR acceptance among Austrian resident doctors. Health Policy Techn. 2015. https://doi.org/10.1016/j.hlpt.2015.02.003.

42. Sedlmayr B, Patapovas A, Kirchner M, Sonst A, Müller F, Pfistermeister B, et al. Comparative evaluation of different medication safety measures for the emergency department: physicians' usage and acceptance of training, poster, checklist and computerized decision support. BMC Med Inform Decis Mak. 2013;13:79. https://doi.org/10.1186/1472-6947-13-79.
43. Pfeiffer Y, Manser T. Development of the German version of the Hospita Survey on Patient Safety Culture: dimensionality and psychometric properties. Safety Sci. 2010. https://doi.org/10.1016/j.ssci.2010.07.002.

44. Neyer FJ, Felber J, Gebhardt C. Development and validation of a brief measure of technology commitment. Diagnostica. 2012. https://doi.org/10. 1026/0012-1924/a000067.

45. Mueller F, Jenny GJ, Bauer GF. Individual and organizational health-oriented readiness for change: conceptualization and validation of a measure within a large-scale comprehensive stress management intervention. Int J Workplace Health Manag. 2012. https://doi.org/10.1108/17538351211268872.

46. Lang G, Stengård E, Wynne R. Developing a scale measuring perceived knowledge and skills dimensions for mental health promotion: a pilot test using a convenience sample. JMHTEP. 2016. https://doi.org/10.1108/ JMHTEP-02-2015-0005.

47. RMHA H i't V, Kosterink SM, Barbe T, Lindegård A, Marecek T, MMR V-H. Relation between patient satisfaction, compliance and the clinical benefit of a teletreatment application for chronic pain. J Telemed Telecare. 2010;16(6): 322-8. https://doi.org/10.1258/jtt.2010.006006.

48. Hölzel LP, Ries Z, Dirmaier J, Zill JM, Kriston L, Klesse C, et al. Usefulness scale for patient information material (USE) - development and psychometric properties. BMC Med Inform Decis Mak. 2015;15:34. https:// doi.org/10.1186/s12911-015-0153-7.

49. Hoffmann B, Domanska OM, Albay Z, Mueller V, Guethlin C, Thomas EJ, et al. The Frankfurt patient safety climate questionnaire for general practices (FraSiK): analysis of psychometric properties. BMJ Qual Saf. 2011;20(9):797805. https://doi.org/10.1136/bmjqs.2010.049411.

50. Haslinger-Baumann E, Lang G, Müller G. Validity and reliability of the "German utilization questionnaire-dissemination and use of research" to measure attitude, availability, and support toward implementation of research in nursing practice. J Nurs Meas. 2014;22(2):255-67. https://doi.org/ 10.1891/1061-3749.22.2.255.

51. Görtz-Dorten A, Breuer D, Hautmann C, Rothenberger A, Döpfner M. What contributes to patient and parent satisfaction with medication in the treatment of children with ADHD? A report on the development of a new rating scale. Eur Child Adoles Psy. 2011;20(Suppl 2):S297-307. https://doi. org/10.1007/s00787-011-0207-z.

52. Ehm JH, Hartmann U, Höltge L, Hasselhorn M. Die Perspektive pädagogischer Fachkräfte auf schulvorbereitende Zusatzförderung in der Kita. Unterrichtswissenschaft. 2017. https://doi.org/10.3262/UW1703239.

53. Becker $D$, editor. Acceptance of mobile mental health treatment applications. 7th International Conference on Emerging Ubiquitous Systems and Pervasive Networks, EUSPN 2016 /The 6th International Conference on Current and Future Trends of Information and Communication Technologies in Healthcare, ICTH-2016/Affiliated Workshops, 2016: Elsevier B.V; 2016.

54. Breimaier HE, Halfens RJ, Lohrmann C. Effectiveness of multifaceted and tailored strategies to implement a fall-prevention guideline into acute care nursing practice: a before-and-after, mixed-method study using a participatory action research approach. BMC Nurs. 2015;14:18. https://doi. org/10.1186/s12912-015-0064-z.

55. Breimaier HE, Heckemann B, Halfens RJ, Lohrmann C. The Consolidated Framework for Implementation Research (CFIR): a useful theoretical framework for guiding and evaluating a guideline implementation process in a hospital-based nursing practice. BMC Nurs. 2015;14:43. https://doi.org/ 10.1186/s12912-015-0088-4.

56. Bradley C, Plowright R, Stewart J, Valentine J, Witthaus E. The Diabetes Treatment Satisfaction Questionnaire change version (DTSQc) evaluated in insulin glargine trials shows greater responsiveness to improvements than the original DTSQ. Health Qual Life Outcomes. 2007;5:57. https://doi.org/10. 1186/1477-7525-5-57.

57. Bott U, Ebrahim S, Hirschberger S, Skovlund SE. Effect of the rapid-acting insulin analogue insulin as part on quality of life and treatment satisfaction in patients with type 1 diabetes. Diabetic Med. 2003;20(8):626-34. https:// doi.org/10.1046/j.1464-5491.2003.01010.x.

58. Zarski AC, Berking M, Fackiner C, Rosenau C, Ebert DD. Internet-based guided self-help for vaginal penetration difficulties: results of a randomized controlled pilot trial. J Sex Med. 2017;14(2):238-54. https://doi.org/10.1016/j. jsxm.2016.12.232.

59. Berger T, Krieger T, Sude K, Meyer B, Maercker A. Evaluating an e-mental health program ("deprexis") as adjunctive treatment tool in psychotherapy for depression: results of a pragmatic randomized controlled trial. J Affect Disord. 2017. https://doi.org/10.1016/j.jad.2017.11.021. 
60. Dreer B, Dietrich J, Kracke B. From in-service teacher development to school improvement: factors of learning transfer in teacher education. Teach Dev. 2017. https://doi.org/10.1080/13664530.2016.1224774.

61. Paulsen HFK, Kauffeld S. Linking positive affect and motivation to transfer within training: a multilevel study. Int J Train Dev. 2017. https://doi.org/10. 1111/ijtd.12090.

62. Beil J, Cihlar V, Kruse A. Willingness to accept an internet-based mobility platform in different age cohorts: empiric results of the project S-Mobil 100. Z Gerontol Geriatr. 2015;48(2):142-9. https:/doi.org/10.1007/s00391-013-0546-0.

63. Kriz D, Nübling R, Steffanowski A, Wittmann WW, Schmidt J. Patients' satisfaction in inpatient rehabilitation. Psychometrical evaluation of the ZUF-8 based on a multicenter sample of different indications. Z Med Psychol. 2008;17(2-3):67-79.

64. Schmidt J, Lamprecht F, Wittmann WW. Satisfaction with inpatient care development of a questionnaire and first validity assessments. Psychother Psychosom Med Psychol. 1989;39(7):248-55.

65. Jerusalem M, Schwarzer R. Skalen zur Erfassung von Lehrer- und Schülermerkmalen. Dokumentation der psychometrischen Verfahren im Rahmen der Wissenschaftlichen Begleitung des Modellversuchs Selbstwirksame Schulen. Berlin: Freie Universität Berlin; 1999. Available from: http://userpage.fu-berlin.de/health/germscal.htm. Accessed: 6 Feb 2018

66. Hinz A, Schumacher J, Albani C, Schmid G, Brähler E. Bevölkerungsrepräsentative Normierung der Skala zur Allgemeinen Selbstwirksamkeitserwartung. Diagnostica. 2006. https://doi.org/10.1026/ 0012-1924.52.1.26

67. Breimaier HE. Implementation of research-based knowledge. Lessons learnt from the nursing practice [dissertation]. Graz: Medical University of Graz; 2015

68. World Health Organization (WHO). Healthy Settings. Types of Healthy Settings. 2018. Available from: http://www.who.int/healthy_settings/types/en/. Accessed: 23 Feb 2018.

69. McDowell I. Measuring health: a guide to rating scales and questionnaires. 3rd ed. New York: Oxford University Press; 2009.

70. Souza AC, Alexandre NMC, Guirardello EB. Psychometric properties in instruments evaluation of reliability and validity. Epidemiol Serv Saude. 2017; 26(3):649-59. https://doi.org/10.5123/s1679-49742017000300022.

71. Haynes SN, Nelson K, Blaine DD. Psychometric issues in assessment research. In: Kendall PC, Butcher JN, Holmbeck GN, editors. Handbook of research methods in clinical psychology. Hoboken, NJ, US: John Wiley \& Sons Inc; 1999. p. 125-54.

72. Schultes MT, Jöstl G, Finsterwald M, Schober B, Spiel C. Measuring intervention fidelity from different perspectives with multiple methods: The Reflect program as an example. Studies in Educational Evaluation. 2015;47:102-112.

73. Champion VL, Leach A. Variables related to research utilization in nursing: an empirical investigation. J Adv Nurs. 1989;14(9):705-10. https://doi.org/10. 1111/j.1365-2648.1989.tb01634.x.

74. Venkatesh V, Davis FD. Theoretical extension of the technology acceptance model: four longitudinal field studies. Manag Sci. 2000. https://doi.org/10. 1287/mnsc.46.2.186.11926

75. Greenhalgh T, Peacock R. Effectiveness and efficiency of search methods in systematic reviews of complex evidence: audit of primary sources. BMJ. 2005;331(7524):1064-5. https://doi.org/10.1136/bmj.38636.593461.68.

76. Danielsen AK, Pommergaard HC, Burcharth J, Angenete E, Rosenberg J. Translation of questionnaires measuring health related quality of life is not standardized: a literature based research study. PLoS One. 2015;10(5): e0127050. https://doi.org/10.1371/journal.pone.0127050.

77. Hoben M, Estabrooks CA, Squires JE, Behrens J. Factor structure, reliability and measurement invariance of the Alberta Context Tool and the Conceptual Research Utilization Scale, for German residential long term care. Front Psychol. 2016;7:133. https://doi.org/10.3389/fpsyg.2016.01339.

78. Sonnentag S, Pundt A. Organisational health behavior climate: organisations can encourage healthy eating and physical exercise. Appl Psychol. 2016; 65(2):259-86. https://doi.org/10.1111/apps.12059.

79. Drzensky F, Egold N, van Dick R. Ready for a change? A longitudinal study of antecedents, consequences and contingencies of readiness for change. J Chang Manag. 2012;12(1):95-111. https://doi.org/10.1080/14697017.2011. 652377.

80. Freund T, Peters-Klimm F, Boyd CM, Mahler C, Gensichen J, Erler A, et al. Medical assistant-based care management for high-risk patients in small primary care practices: a cluster randomized clinical trial. Ann Intern Med. 2016;164(5):323-30. https://doi.org/10.7326/M14-2403.

Ready to submit your research? Choose BMC and benefit from:

- fast, convenient online submission

- thorough peer review by experienced researchers in your field

- rapid publication on acceptance

- support for research data, including large and complex data types

- gold Open Access which fosters wider collaboration and increased citations

- maximum visibility for your research: over $100 \mathrm{M}$ website views per year

At BMC, research is always in progress.

Learn more biomedcentral.com/submissions 